\title{
Determination of Reactivity and Neutron Flux Using Modified Neural Network for HTGR
}

\author{
M. Subekti ${ }^{1 *}$, K. Kudo ${ }^{2}$, K. Nabeshima ${ }^{3}$ and K. Takamatsu ${ }^{4}$ \\ ${ }^{I}$ Center for Nuclear Reactor Technology and Safety, National Nuclear Energy Agency, \\ ,Puspiptek Area Serpong, Tangerang Selatan 15310, Indonesia \\ ${ }^{2}$ Kyushu University, 2-33-19 Mizutani Higashi-ku,Fukuoka 813-0041, Japan \\ ${ }^{3}$ Research Group for Advanced Reactor System, Japan Atomic Energy Agency,Tokai-mura, Ibaraki-ken, Japan \\ ${ }^{4}$ Department of HTTR Project, Japan Atomic Energy Agency, Oarai-machi, Ibaraki-ken 311-1394, Japan
}

\section{ARTICLE INFO}

Article history:

Received 19 November 2014

Received in revised form 24 February 2017

Accepted 17 April 2017

Keywords:

HTTR

Reactivity determination

Method development

Verification

Withdrawal test

Online application

\begin{abstract}
A B S T R A C T
Nuclear kinetic calculations based on point kinetic model have been generally applied as the standard method for neutronics codes. As the central control rod (C-CR) withdrawal test has demonstrated in a prismatic core type high-temperature gas-cooled reactor (HTGR) named High Temperature Engineering Test Reactor (HTTR), the transient calculation of kinetic parameter, reactivity, and neutron fluxes, requires a new method to shorten calculation-process time. Development of neural network method was applied to point kinetic model as the necessity of real-time calculation that could work in parallel with the digital reactivity meter. The combination of Time Delayed Neural Network (TDNN) and Jordan Recurrent Neural Network (Jordan RNN) named TD-Jordan RNN was the result of the modeling approach. The application of TD-Jordan RNN with adequate learning, tested offline, determined results accurately even when signal inputs were noisy. Furthermore, the preprocessing for neural network input utilized noise reduction as one of the equations to transform two of twelve time-delayed inputs into power corrected inputs.
\end{abstract}

\section{INTRODUCTION}

Japan Atomic Energy Agency (JAEA) has constructed a high-temperature gas-cooled reactor (HTGR) as a technology development that could provide electricity generation as well as heat for applications such as hydrogen generation and coal liquefaction. The HTGR has a prismatic core named the High Temperature Engineering Test Reactor (HTTR). The major specifications of the HTTR are shown in Table 1. Safety demonstration tests were conducted to demonstrate the inherent safety features of the HTTR [1]. The central control rod (C-CR) withdrawal test was one of the HTTR safety demonstration tests besides coolant circulator trips. The simulation of power transient employs software simulation and measurements during the withdrawal test. Utilized standard codes such as SRAC, MVP, and RELAP codes require long calculation process

* Corresponding author.

E-mail address: subekti@batan.go.id

DOI: https://doi.org/10.17146/aij.2017.683 time. Therefore, the standard codes are difficult to use for online determination purpose of reactor parameters such as neutron flux and reactivity. Hence, an alternative method is needed. The research presented in this paper attempted to create such a method. It focused on modifying neural network $(\mathrm{NN})$ to create a new method that approximated the point kinetic model.

The fuel temperature coefficient, as one of the reactivity coefficients, will be the most important parameter for reactivity and neutron flux determination during control rod withdrawal. An NN modification approximating the classical point kinetic model of reactor physics has been done and tested for online reactivity determination. In another progress in nuclear power plant monitoring, the condition of the reactor can be effectively monitored by analyzing small fluctuatons in the process variables, such as reactivity coefficients [3,4]. However, in a power transient, a reactor with strong negative reactivity responds to the reactivity insertion by correcting the total 
reactivity. This correction can be done in coupled neutronics and thermalhydraulic calculations. This fast coupled calculation is commonly applied in PWR simulators such as VERA-CS [5], PC-TRAN [6], and BATAN PWR Simulator [7]. In the case of NN application, NN could help the core designer for peaking factor prediction [8]. However, progresses in advanced technologies such as NN technique for prediction nuclear physics parameter have been very rare.

This research aimed at developing a new method for online reactivity and neutron flux determination during C-CR withdrawal as well as pretest analysis of reactivity insertion accident. The development of the new method was carried out by modification of $\mathrm{NN}$ for approximation of classical point kinetic model. The input layer of the $\mathrm{NN}$ is equipped with special preprocessing (SPP) as one of the time delayed Jordan recurrent neural network (TD-Jordan RNN) features, in which the initial power input correlates to the $\mathrm{C}-\mathrm{CR}$ inputs. SPP containing noise reduction transformed two of the twelve time-delayed inputs into two different characterized inputs. Furthermore, the research investigated the extrapolation and interpolation capability.

Table 1. Major specifications of the HTTR [2]

\begin{aligned} & Thermal Power $30 \mathrm{MW} \\ &$ Reactor coolant Helium gas \\ & Outlet coolant temperature $850{ }^{\circ} \mathrm{C} / 950{ }^{\circ} \mathrm{C} \\ &$ Inlet coolant temperature $395{ }^{\circ} \mathrm{C} \\ &$ Primary coolant pressure $4 \mathrm{MPa} \\ &$ Core material Graphite \\ & Core diameter $290 \mathrm{~cm} \\ &$ Average power density $2.5 \mathrm{~W} / \mathrm{cm}^{3} \\ &$ Fuel Low-enrichment $\mathrm{UO}_{2} \\ &$ Fuel element type Prismatic block \\ & Uranium enrichment $3-10 \%$ (av. $6 \%) \\ &$ Pressure vessel Steel (21/4 Cr-Mo) \\ & \hline Number of main cooling system 1 \\ & \hline\end{aligned}

\section{METHODOLOGY}

The research steps consisted of experiments on control rod withdrawal and NN modification approximating classical point kinetic model to determine the reactivity and neutron flux. The global analysis error was presented to study the determination capability of modified NN. For more detail investigation, the $9-15 \mathrm{MW}$ learning data was used for extrapolation test and 9-15-18MW learning data was used for interpolation and extrapolation tests.

\section{Experiment on control rod withdrawal}

In a reactor safety demonstration, C-CR withdrawal at critical condition was assumed as a cause of an early reactivity accident. C-CR was located at the center of the reactor core. A central pair, out of 16 pairs of control rods, was withdrawn and a reactivity insertion event without scram was measured and simulated offline.

Figure 1 shows withdrawal test effects on reactivity and neutron flux with the reactivity transient affected by a C-CR withdrawal of $20 \mathrm{~mm}$. The research conducted measurements during the C-CR withdrawal tests for withdrawals of $20 \mathrm{~mm}, 30 \mathrm{~mm}$, and $40 \mathrm{~mm}$ at power levels of $9 \mathrm{MW}, 15 \mathrm{MW}$, and $18 \mathrm{MW}$, respectively. The online testing was conducted as well at a power level of $24 \mathrm{MW}$. Furthermore, the test also adopted two withdrawal speeds: slow mode (about $1.45 \mathrm{~mm} / \mathrm{s}$ ) and fast mode (more than $3.8 \mathrm{~mm} / \mathrm{s}$ ). Table 2 presents the measurement results of 17 cases of C-CR withdrawal tests. The C-CR withdrawal test for $40 \mathrm{~mm}$ at fast speed was not performed at $15 \mathrm{MW}$ because of the technical limitations; rather, the test used a $10 \mathrm{~mm}$ withdrawal at a similar speed.

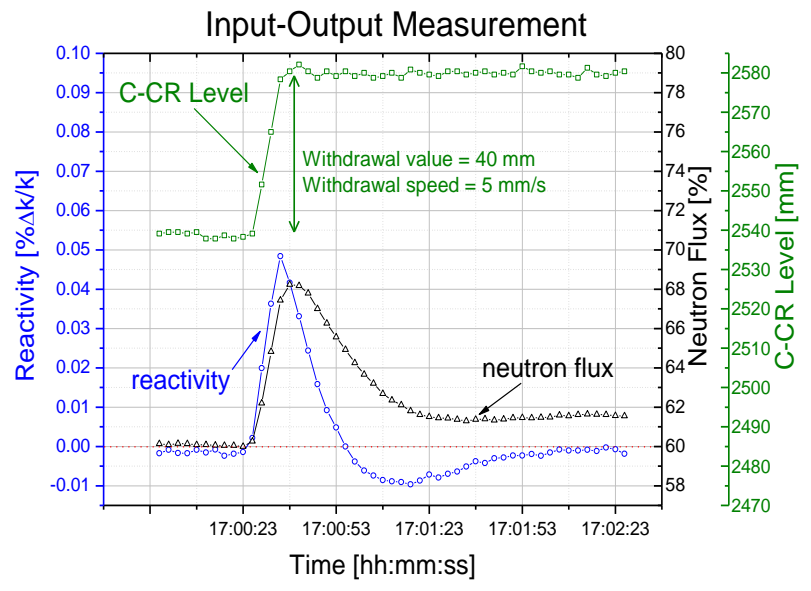

Fig. 1. Withdrawal test effects on reactivity and neutron flux with the reactivity transient is affected by C-CR withdrawal of $20 \mathrm{~mm}$.

In the model, the acquisition time was assumed to be three seconds. The C-CR withdrawal test data at the reactor power of $9 \mathrm{MW}$ was recorded. Furthermore, the results of the same tests for reactor powers of $15 \mathrm{MW}$ and $18 \mathrm{MW}$ were recorded for the acquisition time of one second. Consequently, three data files which had a threesecond acquisition time were generated from the original data file that had a one-second acquisition time.

Globally, all data were stored in 43 files grouped in 17 measurement conditions as shown in Table 2. The calculated withdrawal values and 
withdrawal speeds are presented in this table. The withdrawal values were measured from the difference of C-CR position averages. The experiments performed consisted of slow withdrawals at about $1.47 \mathrm{~mm} / \mathrm{s}$ and fast withdrawals at about $4.44 \mathrm{~mm} / \mathrm{s}$.

Table 2. The measurement results of 17 cases of C-CR withdrawal test.

\begin{tabular}{|c|c|c|c|c|c|}
\hline \multirow[b]{2}{*}{$\begin{array}{l}\text { Condition } \\
\text { Number }\end{array}$} & \multirow[b]{2}{*}{$\begin{array}{l}\text { Data } \\
\text { Name }\end{array}$} & \multirow[b]{2}{*}{$\begin{array}{c}\text { litial Pow } \\
{[\%]}\end{array}$} & \multicolumn{2}{|c|}{ Withdrawal } & \multirow[b]{2}{*}{$\begin{array}{c}\text { Flux } \\
\text { Increase } \\
{[\%]}\end{array}$} \\
\hline & & & $\begin{array}{l}\text { Value } \\
{[\mathrm{mm}]}\end{array}$ & $\begin{array}{c}\text { Speed } \\
{[\mathrm{mm} / \mathrm{s}]}\end{array}$ & \\
\hline 1 & 9MW1 & 29.4 & 20.61 & 1.47 & 2.246855 \\
\hline 2 & 9MW2 & 29.7 & 39.95 & 1.48 & 4.105406 \\
\hline 3 & 9MW3 & 30.1 & 21.38 & 4.64 & 2.513532 \\
\hline 4 & 9MW4 & 30.2 & 39.37 & 4.45 & 4.656803 \\
\hline 5 & 15MW1 & 51.0 & 20.53 & 1.47 & 3.417861 \\
\hline 6 & 15MW2 & 51.1 & 30.76 & 1.47 & 4.850839 \\
\hline 7 & 15MW3 & 51.5 & 41.41 & 1.49 & 5.956258 \\
\hline 8 & 15MW4 & 50.1 & 18.55 & 4.45 & 3.257523 \\
\hline 9 & 15MW5 & 50.2 & 27.99 & 4.35 & 4.995771 \\
\hline 10 & 15MW6 & 51.0 & 28.77 & 4.44 & 4.972806 \\
\hline 11 & 15MW7 & 50.6 & 12.12 & 4.38 & 2.095365 \\
\hline 12 & 18MW1 & 60.2 & 20.30 & 1.45 & 4.004648 \\
\hline 13 & 18MW2 & 60.2 & 30.04 & 1.43 & 5.595942 \\
\hline 14 & $18 \mathrm{MW} 3$ & 59.9 & 40.23 & 1.44 & 6.633646 \\
\hline 15 & 18MW4 & 60.1 & 19.41 & 3.88 & 3.784463 \\
\hline 16 & 18MW5 & 60.1 & 31.39 & 3.92 & 6.312897 \\
\hline 17 & 18MW6 & 60.2 & 41.53 & 4.15 & 8.227152 \\
\hline
\end{tabular}

\section{Nuclear physics approach}

In classical point kinetic calculation of shortterm reactivity, the modeling approaches the effects of reactor feedback for responding to external reactivity insertion. Reactivity is usually a function of time and frequently depends on neutron population $n(t)$. Therefore, the equations are generally nonlinear. Reactivity also depends on flux due to several factors. In the early stages of the process, the power influences the temperature of the components of the reactor core. Doppler effect also causes temperature change that directly affects the macroscopic cross sections and strongly influences reactivity. There are many feedbacks in the reactor, but only certain feedbacks significantly affect reactivity. Therefore, Doppler broadening needs to be determined and incorporated into the solution of the point kinetics equations [9].

Several mathematical techniques have approximated the model of C-CR withdrawal. One such technique is the reactor point kinetic, which describes the reactivity $\rho(t)$ as a sum of two contributions [9]:

$$
\rho(t)=\delta \rho_{e x t}(t)+\delta \rho_{f}(t)
$$

This equation describes the modeling hypothesis for reactivity calculations based on the point kinetic model. Equation (1) shows how the reactivity calculation assumes significant input of $\delta \rho_{e x t}(t)$, which represents external reactivity insertion by adjusting a control rod, and the parameter $\delta \rho_{f}(t)$, which denotes the change in reactivity corresponding to inherent feedback mechanisms. Similarly, another important technique, the perturbation theory, expresses the corresponding change in multiplication in terms of the fluxes characterizing the unperturbed core [9].

The NN application development should perform the input-output modeling of reactivity and neutron flux determination. According to the point kinetic model described in Eq. (1), the effective inputs are external reactivity insertion and inherent feedback. The rod position was the only external reactivity during the withdrawal test. Another effective parameter was the initial power, which was affected by fuel temperature in reactor core. The other feedback mechanisms that were caused by fuel temperature increase were fuel expansion and deformation of Maxwellian spectrum in fuel to higher energy. The fuel expansion for the thermal reactor was small; modeling neglected the parameter correlated with fuel expansion. The deformation of the Maxwellian spectrum was determined by moderator temperature. In fact, during withdrawal, the moderator temperature, flow, etc., increased slowly, and were neglected for the modeling input. In model simplification, the feedback mechanism was triggered by fuel temperature, which correlated with the feedback mechanism. We thus considered only two modeling inputs: control rod position and initial power.

In the organization of the model input, the $\mathrm{NN}$ employed some preprocessing. The control rod position (in $\mathrm{mm}$ ) was converted into the change of the control rod position. The conversion result was dependent on time delay (TD) applied in conversion function formulated by the equation below:

$$
x_{t}=X^{t}-X^{t-n}
$$

where $X$ and $n$ denote C-CR position value (in $\mathrm{mm}$ ) and time-delayed parameter, and $t$ is time (block) for C-CR withdrawal test. One block time equals 3 seconds. The $n$ time variation of 1 to 10 is formed by the TD input characteristics that is described as C-CR position change during power transients. It is shown in Fig. 2. 
The C-CR position change for fast and slow modes of withdrawal is dependent on the aforementioned time delay. The curve of the fast mode withdrawal speed had a higher gradient of increase of C-CR change during withdrawal than shown in Fig. 2. The curve peak height depended on the withdrawal value. Ten TD input used by developed NN covered the effect of withdrawal value until $42.9 \mathrm{~mm}$ if we assumed that the lowest withdrawal value in slow mode was $1.43 \mathrm{~mm} / \mathrm{s}$. The maximum withdrawal value for slow mode in experiment was $41.41 \mathrm{~mm}$, so the use of ten TD inputs was adequate. However, the TD input values of withdrawal may be set to a wider range as well.



Fig. 2. The $n$ time variation that is formed the time delay (TD) input characteristics that is described as the C-CR position change during power transient.

\section{Neural network determination}

The difference of teaching divided by reference signal $d_{j}$ represents the optimum pattern to be performed by the NN as known supervised learning paradigm. The calculated output $Y_{k}$ gives signal error. Hence, the signal error concludes a square error, $E$, by the equation below:

$$
e_{k}=d_{k}-Y_{k}, \text { and } E=\frac{1}{2} \sum_{k=1}^{K} e_{k}^{2}
$$

Thus, learning carries out the update of synaptic weights iteratively with the aim of eventually making the $\mathrm{NN}$ emulate the measured outputs. The weight updates $\left(\Delta w_{j k}{ }^{t}\right)$ at iteration time $t^{\prime}$ are calculated by the delta rule equation below:

$$
\Delta w_{j k}^{t^{\prime}}=-\eta \frac{\partial E^{t^{\prime}}}{\partial W_{j k}^{t^{\prime}}} \text { and } \frac{\partial E^{t^{\prime}}}{\partial W^{t^{\prime}}}=-e_{k}^{t^{\prime}} \varphi_{k}^{\prime}\left(v_{k}^{t^{\prime}}\right) y_{k}^{t^{\prime}}
$$

where $j$ is the input index, $k$ is the output index, and $\eta$ is a constant that determines the learning rate. The weight updates that is assisted by error function iterate the network until it reaches a suitable error.
Therefore, the update of the weights makes the environment knowledge available to the teaching output that is reachable through network iteration number [10]. NN has teachers or reference signals in input-output learning due to the complexity of the model. After an appropriate weight is reached, the forward calculation is faster than an analytical calculation.

We proposed and introduced an improved $\mathrm{NN}$, a combination of Jordan RNN and TDNN, for a special case of reactivity and neutron flux determination during HTTR C-CR withdrawal. The modified Jordan RNN was able to provide Jordan-type feedback by applying TD found usually in TDNN architecture. The modeling exploited the principle of time series input combined with Jordan type feedback. The TD-Jordan RNN was constructed using the modeling approach, considering the input and network model.

Figure 3 shows the architecture of the developed TD-Jordan RNN with SPP. The developed method approximates the fundamental physical concepts governing the behavior of the neutron population in the reactor related with reactivity calculation as shown in Eq. (1).

Jordan RNN and TDNN were the basic contributors to the developed architecture scheme, TD-Jordan RNN. The Jordan RNN received feedback signals from the output layer, close to feedback of neutronic calculation if we refer again to the point kinetic model. Jordan RNN inserted feedback signals from output signals multiplied with a constant value $\gamma$, called the feedback rate, as in the formula below:

$$
x_{i}^{t+1}=\gamma x_{i}^{t}+Y^{t}(1-\gamma), 0 \leq \gamma \leq 1
$$

where $\gamma$ is recurrent parameter, $x_{i}^{t+1}$ is the input of feedback signal at time $(t+1), x_{\mathrm{i}}^{t}$ is the input of feedback signal at time $(t)$, and $Y$ is the network output. The implementation of TDNN to the new scheme was based on C-CR change dependent on some time delay variation in the TD transformation function; the time delay was adjusted by $n$ parameter in range of 1 to 10 . The important feature of TDNN was to make the training dependent on error-prone preprocessing algorithms for time alignment by way of dynamic input based on time-delayed characteristic [10]. Consequently, the previous input made calculations as well as the plant model. The parameter inputs were normalized in $[-1,1]$. Random numbers were set at $30 \%$ of area $[-1,1]$ to smooth errors adequately during early learning; thus, the possibility of getting an undesirable random number was minimal. 


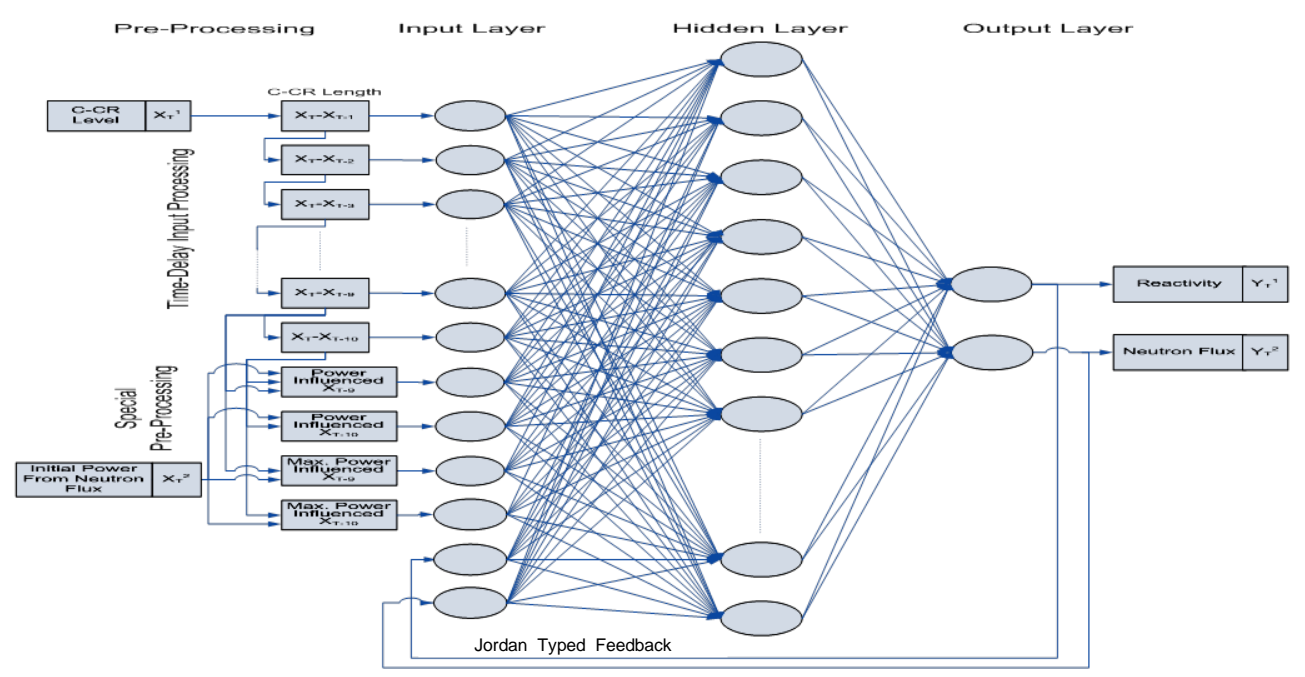

Fig. 3. The architecture of the developed TD-Jordan RNN with special preprocessing.

An important issue to be addressed is how to develop a specialized structure by building prior information into the design [10]. The optimization is the next most important work after modeling by adjusting parameter with some emulation. Verification and validation were performed to ensure that modeling and optimization complied with accuracy requirements. Consequently, optimization found the most accurate processes, such as cross-validation during learning, pattern selection in the class based on nearest neighbor method, and wide pattern selection with new guidelines, for enhancing the extrapolation capability of NN.

\section{Special preprocessing (spp)}

The SPP applied in TD-Jordan RNN estimated input values as the effect of initial power. The feedback was clearly identified after withdrawal, when the measured neutron flux values increased slightly even though the C-CR position changed inputs back to zero. The same level of C-CR position change also gave different neutron flux peak heights depending on the power level. In detail, SPP used initial power values as multiplication factor and maximum function of C-CR position change. SPP enhanced the developed network to follow the power level effect to the feedback mechanism, which affected the reactivity and neutron flux determination.

The SPP contained two advanced preprocessing, $X_{t}^{S P P-1}$ and $X_{t}^{S P P-2}$, at time delays of $T=9$ and $T=10$. For the first SPP $\left(X_{t}^{S P P-1}\right)$, the initial power influenced the maximum function of C-CR position change by using stepped equation starts from $X_{t}^{a}$ calculation using C-CR position change input $\left(x_{t}\right)$. The calculation continued to $X_{t}^{b}$ calculation using $X_{t}^{a}$ input, continued until the last step. $x_{t}$ was corrected by adding $X_{d}$, resulting in $X_{t}^{R A}$ as the desired $\mathrm{NN}$ input.

$$
x_{t} \rightarrow X_{t}^{a} \rightarrow X_{t}^{b} \rightarrow X_{t}^{c} \rightarrow X_{t}^{S P P-1}
$$

Detailed stepped equations are as follows.

- In the noise reduction step, the input is TD C$\mathrm{CR}$ values:

$$
X_{t}^{a}=\frac{X_{t}+X_{t-1}+X_{t-2}+3 X_{t-3}}{6}
$$

- The step for finding maximum value, after noise reduction:

$$
X_{t}^{b}=X_{t}^{a} I f\left(X_{t}^{b}<X_{t}^{a}\right)
$$

- To decrease the acceleration of maximum value and give smoother increase, the next step is:

$$
X_{t}^{c}=\frac{X_{t}^{b}+X_{t-1}^{b}+X_{t-2}^{b} \ldots+X_{t-9}^{b}}{10}
$$

- The multiplication of initial power

$$
X_{t}^{S P P-1}=\frac{P}{100} X_{t}^{C}
$$

where $P$ is the automatically-calculated initial power.

The second preprocessing was a short equation as direct withdrawal multiplication by initial power to the C-CR position, changed as described by the equation (10) below:

$$
X_{t}^{S P P-2}=\frac{P}{100} X_{t}
$$

Equations (9) and (10) appear similar; however, each equation has unique characteristics. 
The first equation results in a smooth increase, as the maximum function describe in eq. (8); the second equation amplifies the multiplication of $\mathrm{C}-\mathrm{CR}$ position change.

\section{RESULTS AND DISCUSSION}

\section{Learning process}

Two general technical terms are used in this study. The first term is pattern generalization to get optimal learned data, and the second term is generalization during learning for learning epoch optimization. Generalization of learned data in a training set is necessary due to the essence of NN learning problem as a curve-fitting problem. The guideline for enhancing the extrapolation capability of $\mathrm{NN}$ is also a pattern generalization method. Another pattern generalization is the nearest neighbor method to select a learned data for each type of data of 15MW1, 15MW3, 15MW5, 15MW7, 18MW1, 18MW3, 18MW4, and 18MW6. The selected data are nearest to neighbored data at the same class of measurement condition.

The learned data contained two pattern data sets. The first pattern data set included 9MW1, 9MW2, 9MW3, 9MW4, 15MW1, 15MW3, 15MW5, and 15MW7 data for NN learning of 9 MW data and 15 MW data. Meanwhile, the $18 \mathrm{MW}$ data was excluded in the first pattern for examining the extrapolation capability of $18 \mathrm{MW}$. Simultaneously, the first pattern was compared to the second in analysis results. The second pattern included 18MW1, 18MW3, 18MW4, and 18MW6 data and a wider pattern selection to assure better extrapolation capability. The test with the first pattern aimed to determine the extrapolation capability when the network had more limited data for learning. Furthermore, we could consider the final result of $9-15-18 \mathrm{MW}$ learning, which had limited data.

The learning optimization should consider that the $\mathrm{NN}$ encodes an input-output mapping into synaptic weights. Consequently, the learning must be extensive enough that generalization to the future is possible. The learning epoch of $10^{5}$ was assumed for performing adequate learning. Figure 4 shows two curves, one pertaining to measurement on the learned data set of $9-15 \mathrm{MW}$ and the other pertaining to the learned data set of 9-15-18MW. The 9-15-18MW learning gave better results as already expected.

Early stopping during learning by identifying the onset of over fitting through the use of crossvalidation assures the best generalization over all learned data sets (learning sets) and tested data sets (validation sets). The cross-validation heuristic suggests that the minimum point of the tested data curve is to be used as a sensible criterion for stopping the learning session [10]. Unfortunately, the cross-validation was unusable, because the optimal epoch for other power levels (less than 9 MW and more than 18 MW) was unknown. The adequate learning was performed by considering the gradient of learning error for stable condition.

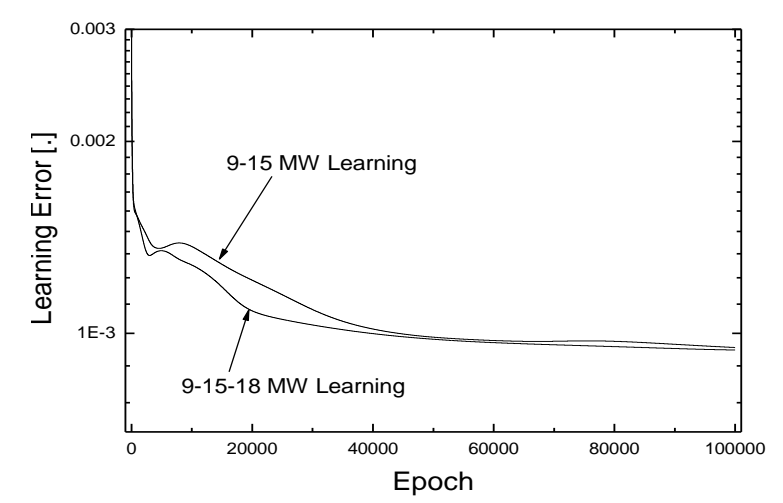

Fig. 4. Learning Error for 9-15 MW learning and for 9-15-18 MW learning.

\section{Analysis result with two different learning}

The calculation process began by entering the C-CR level and initial power. The network determined the reactivity and neutron flux outputs in milliseconds. By using adequate epoch, the determination testing of $9-15 \mathrm{MW}$ and $9-15-18 \mathrm{MW}$ learning were done to all offline data as shown in Fig. 5 for global reactivity analysis error and Fig. 6 for global neutron flux analysis error. The error was the difference between measurement and determination at peak point. The $x$ axis indicates the file name, the condition of which is explained in Table 2. The comparison was made only for the peak point because the significant error was usually recorded in the peak point. Other points may be described by figure.

In reactivity determination, both types of learning had almost the same accuracy characteristics. The significant error of 15MW4-2 data was affected by the error of C-CR position. The reactivity determination test indicated that the network may determine the reactivity at power levels more than $18 \mathrm{MW}$ or less than $9 \mathrm{MW}$. Differently, in neutron flux determination test results, the 9-15-18MW learning yielded slightly better results than 9-15MW learning. Significant error at $18 \mathrm{MW} 6$ data by $9-15 \mathrm{MW}$ learning was due to learning being too poor to generalize a nonlinear condition of 18MW6 data. The test identified the network weakness: the neutron flux determination in extrapolation area resulted in significant errors.

Concerning the significant error in the reactivity determination of $15 \mathrm{MW} 4-2$, Fig. 7 shows 
variation testing of $\mathrm{C}-\mathrm{CR}$ position to understand the possible C-CR position error due to inconsistent withdrawal speed, which may have contributed to the determination error. When withdrawal was finished, the C-CR position data were changed twice by adding $1 \mathrm{~mm}$ and $2 \mathrm{~mm}$ to yield the reactivity determination result change. The first $\mathrm{C}-\mathrm{CR}$ position change resulted in reactivity determination of $0.02536 \% \Delta \mathrm{k} / \mathrm{k}$ (error $=0.00282$ ) and the second C-CR position change resulted in reactivity determination of $0.02709 \% \Delta \mathrm{k} / \mathrm{k}$ (error $=0.00109$ ). The testing error decreased significantly if we made comparisons with the determination result of $0.02368 \% \Delta \mathrm{k} / \mathrm{k}$ (error $=0.00450$ ). All C-CR position input were experiment data without change. Furthermore, the significant error shown in Fig. 5 was unavoidable due to the variance of C-CR position signal from the reactor instrument.



Fig. 5. The global reactivity analysis error.

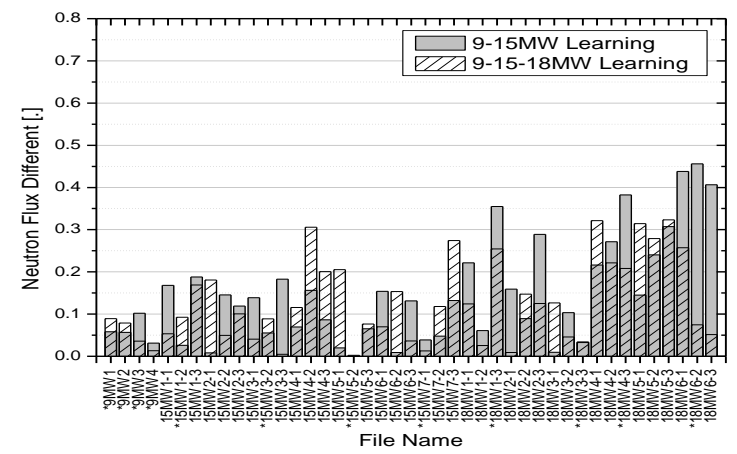

Fig. 6. The global neutron-flux analysis error.

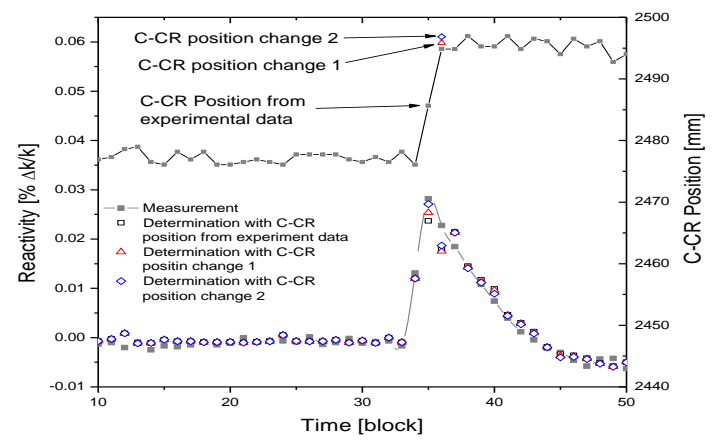

Fig. 7. The error of C-CR position may result in significant error for reactivity determination.

\section{Extrapolation testing}

More detail testing were carried out to show the sensitivity of reactivity and neutron flux determination in point of view of extrapolation and interpolation capability. The results of extrapolation testing for reactivity determination with low withdrawal speed are shown in Fig. 8 and high withdrawal speed in Fig. 9. The low speed mode withdrawal was tested by using 18MW3-2 data and high speed mode withdrawal testing was described by 18MW6-3 data as an example. The TD-Jordan RNN with 9-15MW learning accurately determined the reactivity and neutron flux as well as 9-15-18MW learning. Nevertheless, the TD-Jordan RNN learned 9-15 MW data showed a slight error after withdrawal as shown in Fig. 9. The slight error was due to the Jordan RNN performing the linear learning of $9 \mathrm{MW}$ and 15 MW data. Hence, the 9-15-18 MW learning in an interpolation testing will theoretically improve the $\mathrm{NN}$ for determining the reactivity and neutron flux better than the extrapolation testing. The improved NN will be discussed in the interpolation testing section.

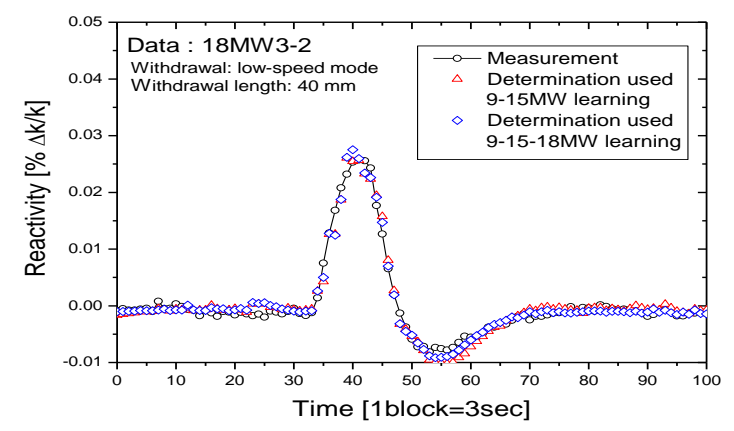

Fig. 8. Extrapolation testing for reactivity determination with low withdrawal speed.

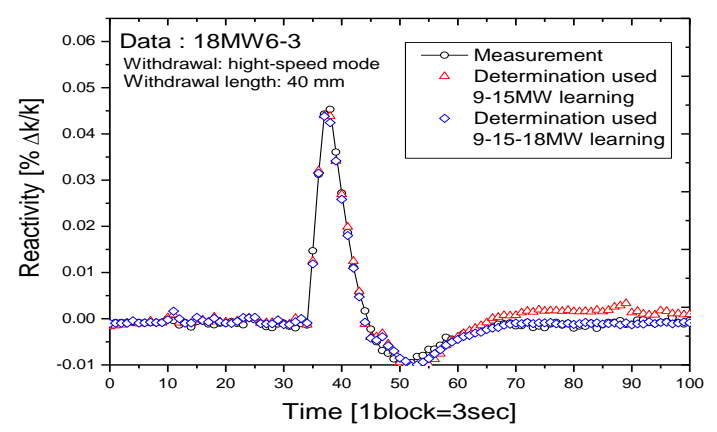

Fig. 9. Extrapolation testing for reactivity determination with high withdrawal speed.

As a follow-up to reactivity determination testing in low and high withdrawal speeds, the results of neutron flux determination is shown in Fig. 10 in low withdrawal speed and Fig. 11 in high withdrawal speed. Result characteristics of both 9-15MW and 9-15-18MW learnings in neutron flux determination were almost similar. The neutron 
flux determinations that are with neither the 9-15MW learning nor 9-15-18MW learning resulted in a good accuracy. However, a slight error occurred in Fig. 11 as well as in Fig. 9, where both tested the high speed withdrawal mode, due to a similar linear problem. The accurate results revealed that TD-Jordan was useful despite linear learning.

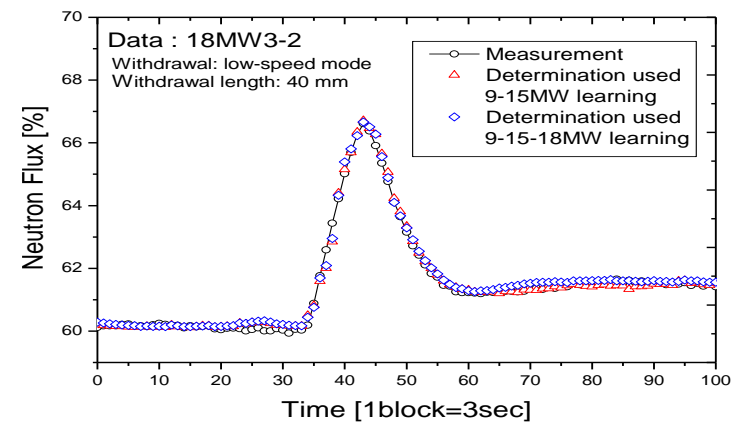

Fig. 10. Extrapolation testing for neutron flux determination with high withdrawal speed.

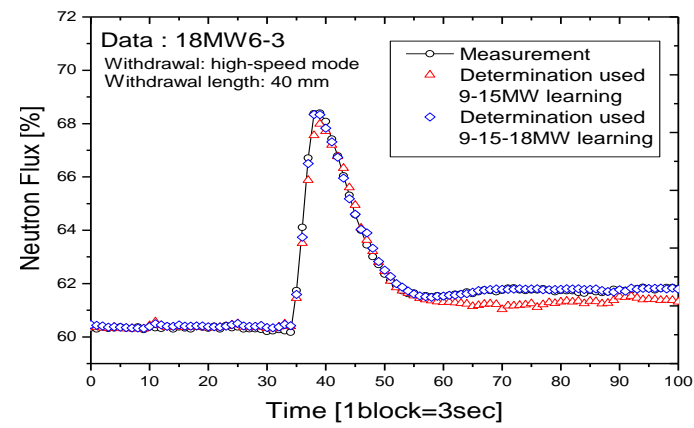

Fig. 11. Extrapolation testing for neutron flux determination with high withdrawal speed.



Fig. 12. Reactivity determination test with low withdrawal speed.

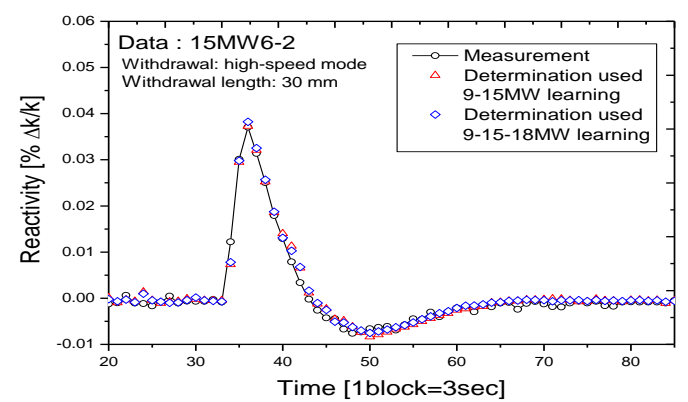

Fig. 13. Reactivity determination test with high withdrawal speed.

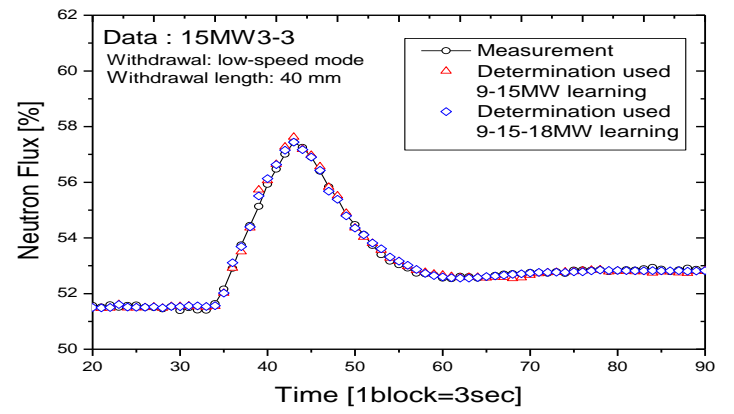

Fig. 14. Neutron flux determination test with low withdrawal speed.

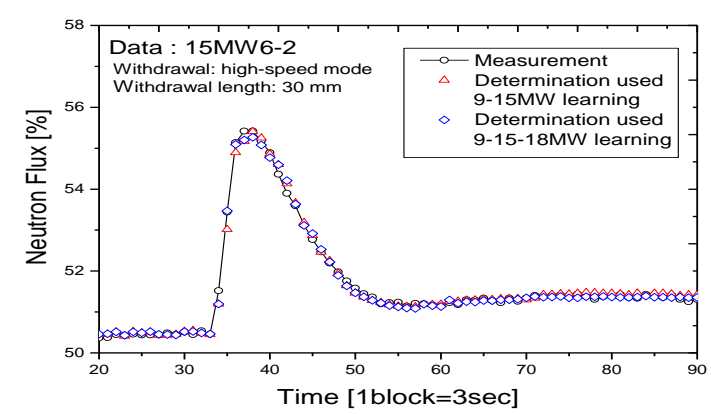

Fig. 15. Neutron flux determination test with high withdrawal speed.

\section{Interpolation testing}

The interpolation testing for reactivity determination were also performed with low and high withdrawal speeds. The interpolation testing results for $15 \mathrm{MW}$ reactor power are shown in Fig. 12 to Fig. 15. The results in these figures show a low error that is similar for other testing using $9 \mathrm{MW}$ or $18 \mathrm{MW}$ data. The 15MW3 data in Fig. 12 and 15MW3-3 data in Fig. 14 are examples for the low speed mode withdrawal. Furthermore, the 15MW6-2 data in Fig. 13 and Fig. 15 are an example for high speed mode withdrawal for both reactivity and neutron flux determination. All examples in the interpolation testing show that the modified NN could give better determination result than extrapolation result, as assumed.

According to the determination error in Fig. 7, potential errors due to the inconsistency of withdrawal speed in fast withdrawal could be solved if the rod level noise divided by withdrawal level is small. However, the other error trend in Fig. 9 and Fig. 11 did not occur in the interpolation case, as the testing results showed in Fig. 13 and Fig. 15.

The testing proved that the developed NN was accurate for modeling and optimization. Adding data in learning improved the NN accuracy, especially in the extrapolation case for getting a merit of nonlinear determination. Hence, learning with limited data created poor knowledge and classimbalance; the unknown class was blindly 
extrapolated. The optimizations that could be taken as learning data preparation required a guideline for enhancing the extrapolation capability, as well as a nearest neighbor method. The learning rate and momentum were stagnant in 0.1 and 0 to assure the stability of a lengthy learning process. A learning of $9-15-18 \mathrm{MW}$ data can take half day to reach 100 thousand epochs with an Intel i3-based computer.

\section{Online testing}

In the TD-Jordan RNN prototype development, the offline and online testing were carried out for the power level of $24 \mathrm{MW}$. Figure 16 shows the online testing utilizing TD-Jordan RNN parallels with other neural network methods of neuro-expert and TSS-MLP [11]. The 9-15-18MW learning data was utilized. All testing results show good prediction with low errors except 24MW1-1 data. Figure 17 shows the extrapolation testing using 24MW1-1 data, showing maximum error prediction. The case of the 24MW1-1 data resulted in larger error than any other cases of $24 \mathrm{MW}$ data. The same problem happened in offline testing for the 18MW6-1 data. The similarity of both data is low withdrawal speed at lowest withdrawal length. This problem is unavoidable due to the outer input-output mapping problem in modified NN. The TD-Jordan that mapped the input-output for reactivity and neutron flux determination found the maximum error at specific data in which the data with higher withdrawal length has been learned more. Consequently, the numerical iteration will give higher error on testing for the data that is learned less than other data. The edge of the mapping fell on the data for the low withdrawal speed at the lowest withdrawal length. However, the maximum error problem only happened on reactivity prediction. The extrapolation testing using 24MW1-1 data shows low error for neutron flux prediction, as shown in Fig. 18.

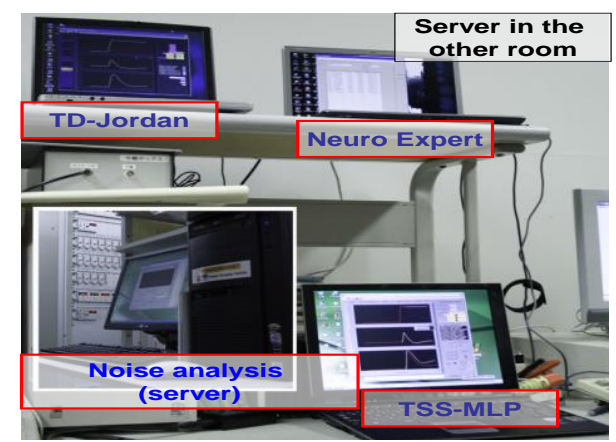

Fig. 16. Online testing utilizing TD-Jordan RNN parallels with other neural network methods of neuro-expert and TSS-MLP [5].

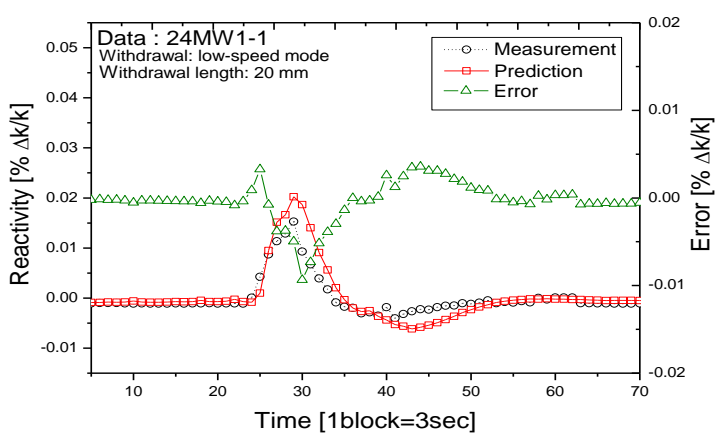

Fig. 17. The extrapolation testing using 24MW6-1 data shows maximum error prediction.

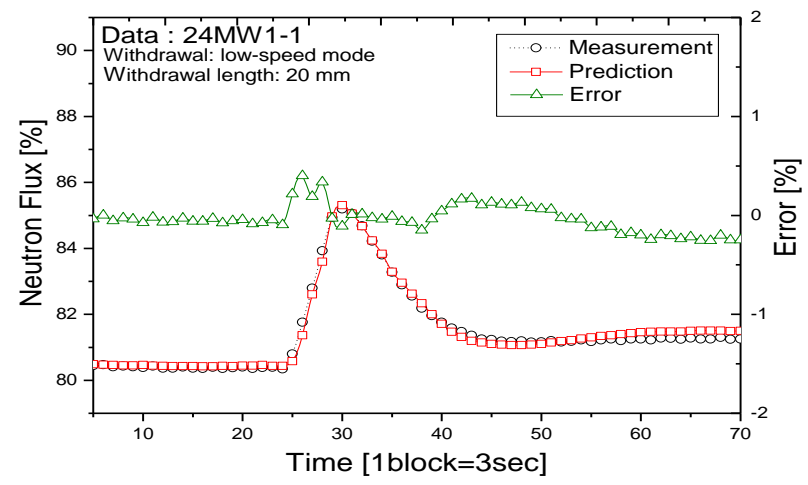

Fig. 18. The extrapolation testing using 24MW6-1 data shows good error for neutron flux prediction.

\section{CONCLUSION}

A modified neural network named TD-Jordan RNN with special preprocessing was developed for reactivity and neutron flux determination. This new method could determine the reactor parameter of reactivity and neutron flux promptly. Therefore, it could be applied in online determination. The TD-Jordan RNN showed good determination accuracy during offline C-CR withdrawal test with reactor power range of 9-18 MW. For best accuracy, TD-Jordan optimization requires wider pattern selection of learning data and learning guidelines for enhancing the extrapolation capability, and the nearest neighbor method was utilized. Furthermore, the online testing were performed to determine the reactivity and neutron flux with power level of $24 \mathrm{MW}$ in which the 9-15-18MW learning data was utilized. The online testing showed accurate results indicated by the difference of measurement and prediction value. However, the maximum error for reactivity determination occurred only for low withdrawal speed at the lowest withdrawal length.

\section{ACKNOWLEDGMENT}

The part of this research also has been conducted as the contract research FY 2002-2007 between Kyushu University and MEXT of Japan. 
We would also like also to thank Dr. Tomio Ohno for technical discussion during the HTTR's experiments.

\section{REFERENCES}

1. K. Takamatsu, X.L. Yan, S. Nakagawa et al., Nucl. Eng. Des. 271 (2014) 379.

2. S. Takada, K. ligaki, M. Shinohara et al., Nucl. Eng. Des. 271(2014) 472.

3. S. Dulla, M. Nervo and P. Ravetto, Ann. Nucl. Energy 65 (2014) 433.

4. H.M. Hashemian, Progress in Nuclear Energy 53 (2011) 167.

5. S. Palmtag, K. Clarno, G. Davidson et al., Coupled Neutronics and Thermal-Hydraulics Solution of A Full-Core PWR Using VERA-CS, Proceedings of International Conference on Physics of Reactors (PHYSOR) 2014, Kyoto, Japan (2014) 1. DOI: http://dx.doi.org/ 10.11484/jaea-conf-2014-003.
6. S.J. Ibrahim, D.R. Ewim and O.A. Edeoja, Leonardo Electronic Journal of Practices and Technologies 22 (2013) 93.

7. M. Subekti, D. Isnaini and E.P. Hastuti, The Determination of Radial Fuel Temperature Distribution in PWR-NPP Simulator on Steady State, Proceeding for Scientific Meeting and Presentation on Basic Nuclear Science and Technology, Book I (2012) 76. (in Indonesia)

8. L.P. Filho, K.C. Souto and M.D. Machado, Using Neural Network for Predicion of Nuclear Parameter, Proceeding for 2013 International Nuclear Atlantic Conference, (2013) 1.

9. M.S. El-Genk and J.M.P. Tournier, Progress in Nuclear Energy 82 (2016) 91.

10. M.T. Hagan, H.B. Demuth, M.H. Beale et al., Neural Network Design, Second Edition, Amazon, US (2014) 7.

11. T. Ohno, M. Subekti, K. Kudo et al., Transactions of the Atomic Energy Society of Japan 4 (2005) 115. 